\title{
SLOW GROUND MOTION MODELLING OF DIAMOND
}

\author{
J. K. Jones, ASTeC, Daresbury Laboratory, Warrington WA4 4AD, UK
}

\begin{abstract}
The need for high stability in third generation light sources has produced strict requirements on the design of the foundations. The specification for the concrete floor stability is a critical task, which can have a major cost impact. A careful assessment of the effects of slow ground motion on the accelerator is therefore required. This paper presents the results of simulations using various ground motion models for the DIAMOND storage ring and the booster synchrotron. The modelling is implemented in flexible manner through the use of a Mathematica to MAD interface. This interface provides various high level functions such as access to an SVD algorithm, which provides effective correction of the closed orbit distortions, the implementation of motion on girders and the statistical analysis and graphical presentation of results.
\end{abstract}

\section{INTRODUCTION}

The DIAMOND project consists of a $3 \mathrm{GeV}$ electron storage ring with a full-energy booster synchrotron injector [1]. The storage ring is designed with an aggressive $2.7 \mathrm{~nm}$-rad emittance, with an emittance ratio of $1 \%$. Under such conditions the long-term stability of the electron beam is essential to maintaining good photon beam quality for users. The stability of the electron beam is generally separated into two regimes; stability over timescales of a few seconds or less, and stability over the timescale of a few seconds to a few months. The most destructive and unpredictable effect on the stability in the longer timescale regime is that due to the motion of the ground underlying the accelerator complex. In the initial phases of machine construction and operation, ground motion due to settling of the newly loaded ground will be dominant. After this time, experience from many accelerators shows that the magnitude of the ground motion decreases to a constant magnitude that continues for the life of the accelerator.

Assuming a perfectly aligned accelerator initially and excluding the short term effects of creep, misalignment of accelerator elements relative to the design orbit grows with time by a random walk process, this is the basis of the ATL model. The misalignment of accelerator elements resulting from this eventually leads to limitations on the operation of the machine, for example because of limits on maximum corrector strengths used to compensate for this. Once the magnitude of ground motion induced displacement of elements is sufficiently large, a re-survey and re-alignment of the accelerator back to some, possibly different, nominal required orbit is undertaken. The frequency of this re-alignment is directly influenced by the rate of growth of element misalignments the sensitivity of the accelerator to such motions, and the corrector strength available.

Since it is only differential motion of accelerator elements that are problematic, the electron beam sensitivity to ground motion vibrations can be reduced by use of a single cast floor, thus increasing the correlated element motion and reducing the differential motion.

The use of girders to mount accelerator elements leads to locally correlated motion. Although girder assemblies can amplify the effects of ground motion in some frequency ranges, in the slow ground motion regime the correlated motion reduces the harmful differential motion of the magnetic elements.

Analytic models can be used to assess the effects of slow ground motion on the alignment of the accelerator and the effect on electron beam properties. Several of these models have been used to assess the effects of slow ground motion on the DIAMOND storage ring and booster synchrotron. In the case of the DIAMOND accelerators however, re-survey and re-alignment intervals have been specified, from which the maximum allowable motion of the magnetic elements and so the floor can be specified. The simulations must therefore produce a specification for floor stability that is required so that ground motion does not limit the machine performance within the specified re-alignment interval.

\section{METHODS FOR SIMULATING GROUND MOTION}

Pure simulation tools for analysing the floor motion of DIAMOND can be split into two regimes; the effects of (a) plane wave motion and (b) diffusive motion. However, at a specific instance in time a diffusive model can be thought of as a superposition of many planar waves of differing phases and amplitudes. Plane wave motion is then a specific case of a diffusive motion model.

The two methods used for modelling the DIAMOND floor are (a) diffusive motion ATL model, and (b) planar wave Fourier and Gaussian model. These are described below.

\section{ATL Diffusive Motion Model}

The ATL model for ground motion is based on empirical evidence collected at many different sites around the world. The ATL model can be thought of as a first order expansion of the real ground motion. This model describes the diffusive or Brownian motion that unconnected points on the ground (or structures attached to the ground) undergo which are uncorrelated and act as a low intensity baseline to the regular (in time and space) motion due to propagating waves in the upper Earth's 
crust. According to this empirical model, the variance of the relative motion of two points scales as the product of the spatial and temporal interval between measurements. The proportionality constant A gives the magnitude of the motion, and may be different at every site as it depends strongly on local ground conditions. The value of A is usually given in $\mu \mathrm{m}^{2} /(\mathrm{m} . \mathrm{s})$, although this can be converted to a $\sigma$ value of ground motion in $\mu \mathrm{m} / 10 \mathrm{~m} /$ year, which is preferred by the project architects.

Applying the ATL model to a circular accelerator is a non-trivial task. Previous work on modelling ATL ground motion was applied to the linear accelerator case. The method for application of ATL to the circular accelerator case has been derived by N. Walker and A. Wolski [2], and is based around the use of co-variant matrices. In essence, the method overcomes the linear distance problem by applying a magnitude of motion to each point based on its variance to every other point in the accelerator. Representative ATL motion can be seen in Figure 1.

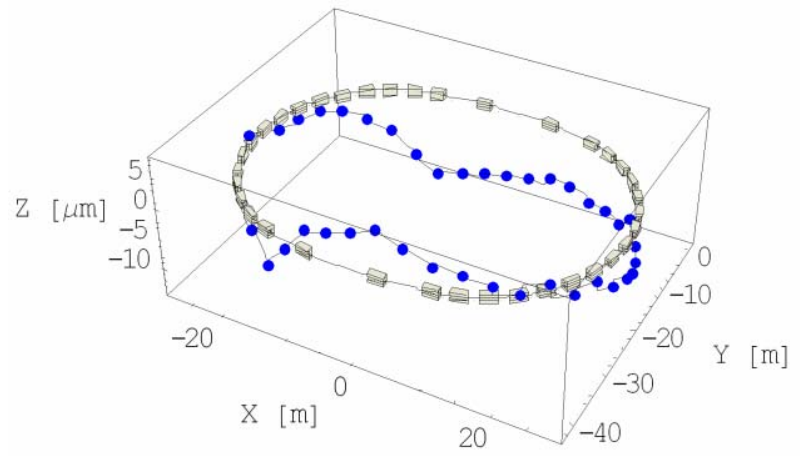

Figure 1: Representative ATL motion over 6 months, with an A factor of $10^{-7} \mu \mathrm{m}^{2} /(\mathrm{m} . \mathrm{s})$, in the DIAMOND booster synchrotron. Quadrupole positions are highlighted as blue points

Due to the diffusive nature of the ATL model, short range differential motion can develop to be unrealistically large, although it is semi-bounded by the magnitude of the A proportionality constant. To ensure a more realistic distribution of motion to the elements, it is important to apply the motion to the magnet girder assemblies. This limits the differential motion between adjacent elements whilst still allowing long range differential motion to occur. It is also a triviality to ensure that the magnets are rotated upon the girders.

\section{Fourier and Gaussian Motion Models}

Modelling the slow ground motion as either a Fourier or Gaussian distributed error is useful for the situations where floor deformations are correlated over long distances.

Relative motion of accelerator elements can be decomposed into a set of Fourier harmonics, with the lowest order harmonic being a tilt in the machine axis. By analysing these harmonics individually one can see the relative effects on the accelerator of different frequencies of ground motion. This can then be compared to known ground motion at the chosen site, giving an insight into the realistic sensitivity of the machine.

Gaussian distributed errors are a special case of applied ground motion, that deals only with the case of localised deformations in the underlying ground. A Gaussian distributed error can apply in superposition with other ground motion errors.

Both of these error distributions are shown in Figure 2.

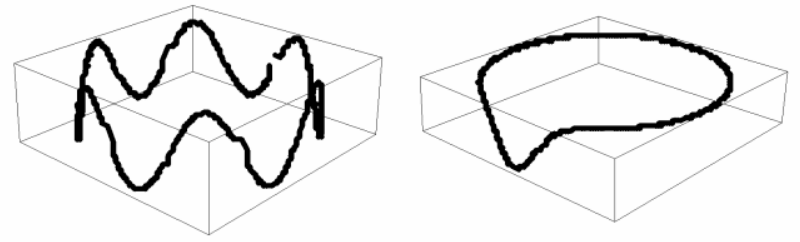

Figure 2: $6^{\text {th }}$ order Fourier distributed error (left) and a $120 \mathrm{~m}$ wide Gaussian distributed error (right) around the DIAMOND storage ring.

\section{COMPUTER SIMULATIONS}

All of the simulations of ground motion modelling are performed through the Mathematica programming environment. A flexible interface to the MAD accelerator code [3] has been written that allows a close integration with the Mathematica environment. Within this Mathematica-to-MAD package, lattices can be defined and modified, using the functions internal to Mathematica. All of the mathematical modelling of ground motion errors is performed within Mathematica, and then the element displacements are written to a MAD deck and the effects on the lattice dynamics calculated.

By using the high-level functionality of Mathematica environment, it becomes trivial to apply element displacements to sets of elements on girders, and to ensure that the girder is tilted and rotated as required. This enables a much more realistic error model to be applied to the accelerator.

The interface between MAD and Mathematica allows functionality to be added to the MAD program without the need to modify the original source code. SVD correction of the closed orbit error is one such functionality that has been added. By using MAD to calculate the BPM response matrices, the mathematical functions within Mathematica then make it easy to invert the matrix and to enable powerful manipulation of the results. The original lattice can then be re-written with newly redefined corrector strengths, and MAD used to calculate the new closed orbit.

As all of the lattice information, and the results of simulations, is available within the Mathematica environment, visualisation of data becomes much simpler. Operations that require basic lattice information, such as lengths or strengths, can be accessed without the need to call MAD, and can be accessed without user intervention. This automation greatly simplifies the analysis of data and can also allow increased complexity of models without a proportional increase in effort. 


\section{STORAGE RING MODELLING}

The DIAMOND storage ring has two correction schemes. The first is for the fast motion of the beam, and features 96 combined horizontal and vertical corrector magnets, two located either end of each long straight section. The second scheme is used to address all motion occurring over periods of greater than seconds, including slow ground motion. The slow correction scheme consists of 168 combined correctors, located in the sextupole magnets. The slow corrector magnets have a specified maximum deflection of $0.8 \mathrm{mrad}$. Previous work has shown that $0.3 \mathrm{mrad}$ of this specification should be allowed to correct static alignment tolerances of magnets upon the magnet girder assemblies. With some contingency, this leaves $0.3 \mathrm{mrad}$ available for slow ground motion correction.

The results of applying varying magnitudes of ATLlike ground motion on the storage ring is shown in Figure 3.

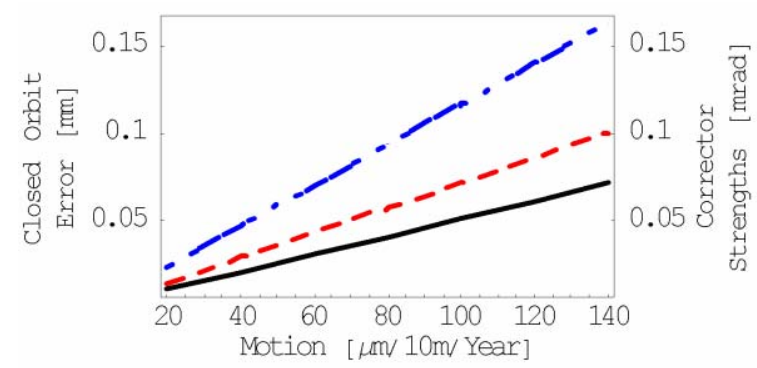

Figure 3: Corrector strength requirements (dashed) and closed orbits at the dipole (solid) and insertion devices (dot dashed) source points in the DIAMOND storage ring under the influence of varying magnitudes of ATL-like ground motion.

The results in Figure 3 show large electron beam offsets at the photon source points. Since this offset is corrected, further minimisation of the electron beam motion at these points would increase the closed orbit error in the rest of the machine, potentially leading to decreased lifetime effects. The diffusive motion inherent in the evolution of these errors would also require an undesirable number of realignments of optical components. For these reasons a specification of $120 \mu \mathrm{m} / 10 \mathrm{~m} /$ year for the storage ring floor motion has been chosen.

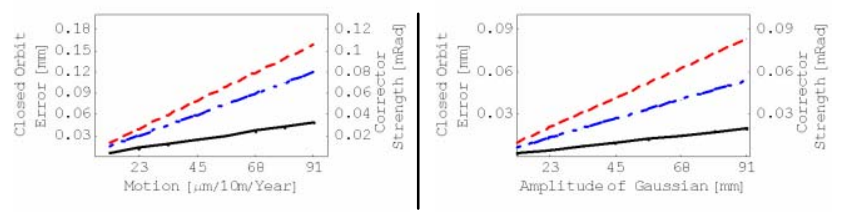

Figure 4: Corrector strength requirements (dashed) and closed orbit at the dipole (solid) and insertion devices (dot-dashed) in the DIAMOND storage ring under the influence of Fourier (left) and Gaussian (right) ground motion.
With the Fourier and Gaussian error models, the storage ring lattice is more sensitive to Fourier errors. This is shown in higher corrector requirements.

The analysis, shown in Figure 4, leads to a ground motion specification of $100 \mu \mathrm{m} / 10 \mathrm{~m} /$ year based upon residual closed orbit error specifications.

\section{BOOSTER MODELLING}

The DIAMOND booster synchrotron includes 22 horizontal and 22 vertical steering magnets, with 22 combined H/V BPMs.

Currently the booster apertures include an allowance of up to $1.4 \mathrm{~mm}$ for the effects of ground motion and other factors not already included. The ground motion effects are directly influenced by the choice of dynamically ramped correctors, or static correction. In the case of static correction at injection energy, closed orbit errors at $3 \mathrm{GeV}$ will be significantly larger, and therefore floor specifications much tighter.

Inclusion of the effects of ATL, Fourier and Gaussian errors gives a specification for the allowable ground motion of greater than $500 \mu \mathrm{m} / 10 \mathrm{~m} /$ year with dynamic correction. With only static correction this drops to much less than $100 \mu \mathrm{m} / 10 \mathrm{~m} / \mathrm{year}$, and leads to an increase in the defined booster apertures. This can be partly overcome by performing re-alignment of the machine more often than the 6 month interval the specification currently calls for.

\section{CONCLUSIONS}

Due to the strong-focusing nature of the DIAMOND storage ring, the contribution of slow ground motion on the degradation of beam optics is considerable. The accelerator floor plays an important role in damping the underlying ground motion and so has significant effects in determining the interval between re-survey of the machine, and so the absolute long term stability of the electron and photon beams. Modelling of the floor motion as diffusive random displacements has lead to a floor specification that should enable the DIAMOND storage ring to operate within its long term stability goals for the specified 6 monthly periods between re-alignments.

The requirements on the booster floor motion are less stringent than the storage ring. The main limitation is the requirement to maintain less than $1.4 \mathrm{~mm}$ of closed orbit error, due to aperture restrictions. Under the assumption of dynamically ramped correctors the specification given is consistent with a 6 monthly re-alignment of the machine.

\section{REFERENCES}

[1] V P Suller, "Status Of The Diamond Light Source Project", EPAC'02, Paris, June 2002.

[2] Andrzej Wolski, Nicholas Walker, "A Model of ATL Ground Motion for Storage Rings", these proceedings.

[3] H. Grote and F.C. Iselin, "The MAD Program", CERN/SL/90-13 (1995). 